\title{
Análisis del Comportamiento de un Chorro Turbulento en un Recinto Cerrado
}

\author{
Carmen Viñas $^{1}$, Gregorio Maqueda ${ }^{2}$, Begoña Blasco ${ }^{1}$ y Juan-Mario García ${ }^{3}$ \\ (1) Universidad Politécnica de Madrid, E.U. de Arquitectura Técnica, Departamento de Tecnología \\ de la Edificación, Avda. de Juan de Herrera 6, 28040 Madrid-España. \\ (e-mail: carmen.vinas@upm.es, begona.blasco@upm.es) \\ (2) Universidad Complutense de Madrid, Facultad de Ciencias Físicas, Departamento de Física de la \\ Tierra Astronomía y Astrofísica II, Avda. de la Complutense s/n, 28040 Madrid-España. \\ (e-mail: gmaqueda@fis.ucm.es) \\ (3) Universidad Politécnica de Madrid, E.U. de Ingeniería Técnica Industrial, Departamento de Física \\ Aplicada, Ronda de Valencia 3, 28012 Madrid-España (e-mail: juanmario.garcia@upm.es)
}

\begin{abstract}
Resumen
Se estudia la estructura de un chorro no isotérmico introducido en el interior de una celda de geometría sencilla, que representa una habitación a escala real, y el intercambio de calor por transporte turbulento asociado al chorro. Utilizando una base de datos de velocidad obtenida con anemómetros sónico y de hilo caliente, así como de temperatura con termopares, se calcula el número de Arquímedes (Ar), la anchura térmica del flujo $\left(I_{m}\right)$ y los números adimensionales de Rayleigh (Ra) y Nusselt (Nu). Se determina la intensidad de turbulencia y los flujos de momento y calor, realizando el análisis de la varianza con el objetivo de contrastar la dependencia considerada, entre estos parámetros, según criterios de significación estadística. Los resultados muestran el comportamiento de las fuerzas de origen térmico y de inercia están asociados al chorro y al transporte de calor de origen turbulento en el interior de la celda.
\end{abstract}

Palabras clave: chorro, flujos de momento y de calor, longitud térmica, número de Arquímedes, número de Nussetl.

\section{Analysis of the Behavior of a Turbulent Jet Indoor}

\begin{abstract}
The structure of a non-isothermal jet coming into a single cell, or room of actual scale, has been studied, as well as the exchange of turbulent heat related to the jet. Using a data base of velocity by sonic and hot-wire anemometers and temperature measured by thermocouple, they are calculated several characteristic parameters of the flow: the non-dimensional numbers Archimedes (Ar), Rayleig $\mathrm{Ra}$ ) and Nusselt (Nu) and the thermal wide of the flow. The intensity of turbulence and the momentum and heat fluxes have been obtained by means of an analysis of variance in order to test the dependence among these parameters, all performed under statistical criteria. Results show the behaviour of the strength of thermal origin and from inertia associated to the jet and the turbulent transport of heat into the cell.
\end{abstract}

Keywords: jet, thermal length, moment and heat fluxes, Archimedes number, Nusselt number 


\section{INTRODUCCIÓN}

Un chorro es el tipo de flujo más importante que se presenta en ventilación de edificios o habitaciones y se define como la descarga de un fluido desde una abertura al interior de un recinto en donde, claramente, está definida una velocidad distinta a la de sus alrededores (Etheridge y Sandberg, 1996). Un chorro cuya temperatura es igual a la temperatura ambiente de la habitación sobre la que incide, se llama chorro isotérmico; por el contrario cuando la temperatura del chorro es diferente de la temperatura ambiente de la habitación se habla de chorro no isotérmico. En mecánica de fluidos a esta clase de chorros se les conoce como chorros de flotabilidad, debido a que en ellos el momento y la flotabilidad, que depende de la distribución de densidades, están presentes.

En los flujos de aire que se presentan en el interior de una habitación se pueden distinguir dos tipos: flujo primario y flujo secundario. Flujo primario es el aire que penetra en el recinto e interacciona con el aire ambiente (Kotsovinos y Angelidis, 1991; Launder y Rodi, 1983). Se origina, así, un flujo secundario de velocidad menor que la del flujo primario, pero ocupando una mayor extensión que éste en el volumen de la habitación. Ambos tipos de flujos pueden tener carácter turbulento, a no ser que en el flujo secundario la flotabilidad y las bajas velocidades se den al mismo tiempo.

Cuando un chorro de aire se introduce en una habitación a una temperatura superior a la del aire ambiente, es posible que el aire circule solamente en las zonas próximas al techo, produciéndose una estratificación del flujo en aquellas zonas ocupadas por el mismo y dando lugar a pequeñas variaciones en la transferencia de flujo de calor dentro de la habitación (Dubovsky et al, 2001). Por el contrario, si el chorro de aire que se introduce es un chorro de aire frío, éste tiende a expansionarse alcanzándose velocidades relativamente altas, lo que implicará una variación significativa en la transferencia de calor dentro de la habitación. Por tanto, es importante para el estudio de un chorro en una habitación, conocer sus campos de velocidad y de temperatura.

Inicialmente cuando una masa de aire es introducida en un recinto o habitación predominan en mayor medida las fuerzas de inercia del aire. Debido a que dicha masa de aire puede ser más o menos densa que el aire ambiente, las fuerzas de flotabilidad pueden ganar importancia, al cabo de un cierto tiempo. Este hecho hace que la relación entre el flujo de momento y el flujo de flotabilidad sea de gran importancia para el comportamiento de un chorro (Costa, 1999). Dado que este proceso está altamente influenciado por la naturaleza turbulenta del flujo es esencial un estudio completo, de dichos flujos, a la hora de diseñar los sistemas de ventilación de un edificio.

El objetivo de este trabajo, es presentar por un lado, el análisis de la influencia de los flujos de momento y de calor, de naturaleza turbulenta, sobre la agitación turbulenta de un chorro, no isotérmico, introducido en una celda de geometría sencilla que representa una habitación a escala real. Por otro lado, se analizar la dependencia del intercambio de calor en el interior de la celda con las fuerzas de origen térmico y de inercia asociadas al chorro.

\section{METODOLOGÍA}

Se ha utilizado la base de datos de velocidad y de temperatura generada en un modelo experimental (Viñas, 2003; Viñas y Maqueda, 2005). Para el desarrollo del modelo se utilizó una celda de geometría sencilla, que representa una habitación a escala real, cedida por el Instituto de Energías Renovables del CIEMAT en el Laboratorio de Ensayos Energéticos para Componentes de la Edificación de la Planta Solar de Almería (España).

Básicamente el modelo consistió en la realización de mediciones de velocidad y temperatura del aire mediante la formación de una malla de sensores en su interior. Para la medida de la velocidad se utilizan 6 anemómetros de hilo caliente (HC), un anemómetro sónico (AS) que además da temperaturas y 8 termopares (TP) para la medida de esta última magnitud. Los TP y AHC tienen tasas de muestreo de $1 \mathrm{~Hz}$ y estaban recientemente calibrados, el AS posee una tasa de muestreo de $21 \mathrm{~Hz}$. 
El aire se introdujo en la habitación de ensayos, de forma controlada mediante un motor compresor, a través de una ventana de dimensiones $0.55 \mathrm{~m} \times 0.57 \mathrm{~m}$ situada en la pared Sur de la celda y próxima al plano central de la habitación (Fig. 1). El flujo de aire introducido con la ayuda del compresor, en la celda, se hizo a través de un tubo flexible conectado por un extremo al compresor y por el otro a un dispositivo en forma de tronco de pirámide que adaptaba el flujo al tamaño de la ventana manteniendo, aceptablemente, el carácter axil-simétrico original de la descarga de aire, y en el que, además, se colocaron dos rejillas para disminuir el efecto de las posibles perturbaciones del compresor.

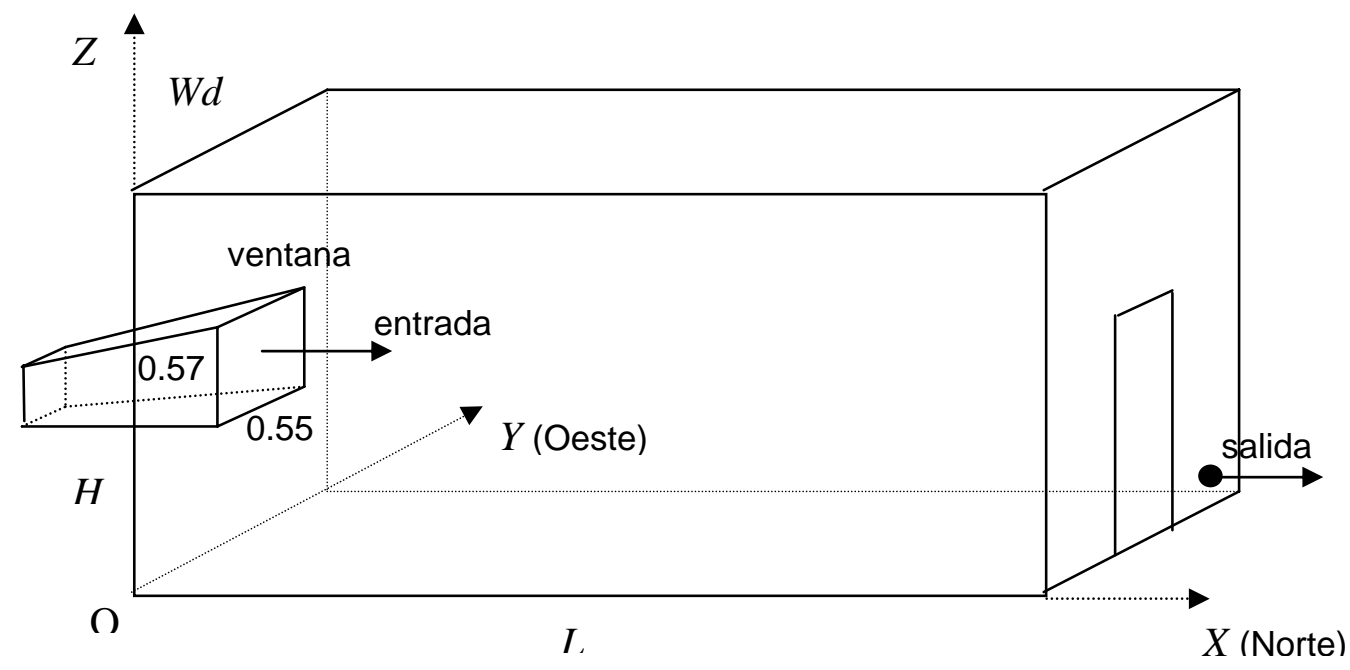

Fig.1: Esquema de la habitación de ensayos, donde se ha marcado la posición de la ventana de entrada de aire en la pared sur. La salida se encuentra en la pared norte cerca de la puerta de acceso (cerrada durante las medidas). Dimensiones: largo $(L): 5.45 \mathrm{~m}$; ancho $(W d): 2.96 \mathrm{~m}$; alto $(H): 2.76 \mathrm{~m}$. Ventana: $0.55 \times 0.57 \mathrm{~m}^{2}$.

La salida del aire del recinto de la celda es un orificio circular situado en la cara Norte de la misma, de área menor que la de la entrada, pero suficientemente grande para que el aire saliera sin dificultad. Cinco anemómetros de hilo caliente se colocaron en un soporte vertical junto a cinco termopares, todos ellos espaciados regularmente para obtener perfiles verticales de temperatura y velocidad. El anemómetro sónico se dispuso a una altura intermedia de los sensores anteriores detrás de ellos, a una distancia de $50 \mathrm{~cm}$, y orientado en la dirección general del flujo. Así mismo, se colocó un AHC y un TP en el centro de la ventana, un TP en la pared Este y otro en la pared Oeste respectivamente que sirvieron para la obtención de temperaturas de referencia.

La formación de la malla se llevó a cabo en tres fases: fase A, los perfiles cubren toda la altura de la habitación (niveles: $0.45,0.90,1.35,1.80,2.25 \mathrm{~m}$ ); fase $\mathrm{B}$, con perfiles desde el suelo hasta $1.25 \mathrm{~m}$ y fase $C$, con perfiles muy próximos al suelo (nivel máximo a $0.75 \mathrm{~m}$ ). En cada una de ellas, mediante el desplazamiento a distintos puntos de la habitación del soporte vertical con los AHC y TP (X: 1.5, 2.5 y 3.5 m; Y: 0.4, 0.9, 1.48, 2.06, 2.56 y 2.76 m), junto con el anemómetro sónico, se formó la malla. La longitud de cada registro (15 minutos) es suficientemente larga para alcanzar el régimen estacionario y, a la vez, evitar tendencias en la temperatura de entrada. En la elaboración del trabajo se han utilizado diferencias de temperatura respecto a la medida en la pared Este. Un total de 18 posiciones de la columna de sensores fueron empleadas para cubrir el volumen de la habitación (fase A). Esto representa una malla tridimensional de 90 puntos con AHC y TP, además de 18 con AS en un plano a $1.43 \mathrm{~m}$ sobre el suelo. En las fases B y C las posiciones fueron 12 cubriendo sólo la mitad Oeste de la celda, y con el AS a 0.7 y $0.43 \mathrm{~m}$ de altura respectivamente.

Previamente a cada una de las fases del proceso experimental, se realizaron medidas de velocidad con el AS a la entrada de la ventana, en distintas posiciones, lo que permitió conocer su distribución justo a la entrada, que dío como resultado un perfil prácticamente parabólico característico de chorros con simetría circular. Del perfil obtenido se calcula el caudal aportado al recinto considerando una expansión del chorro en forma cónica (Lauder y Rodi, 1983), en donde la velocidad media del 
chorro para una sección dada puede aproximarse por: $\bar{V}=V_{\max } / 2$, donde $V_{\text {max }}$ es la velocidad máxima. El valor de referencia de esta velocidad en los experimentos es $2 \mathrm{~ms}^{-1}$. Durante todos los ensayos se midió la velocidad en el centro de la ventana con un AHC, no observándose variaciones significativas respecto a las medidas previas.

Los instrumentos de medida de temperatura y velocidad utilizados han permitido una alta resolución temporal en los datos, facilitando el cálculo de flujos de calor (Kruse y von Rohr, 2006) y momento, así como de intensidad de turbulencia (Costa et al., 1999). A través de un programa preparado al efecto se calcularon los flujos de momento en todos los puntos de la malla, a partir de la determinación de las covarianzas $\overline{u^{\prime} u^{\prime}}, \overline{u^{\prime} v^{\prime}}, \overline{u^{\prime} w^{\prime}} \ldots$, lo que constituye el tensor de Reynolds

$$
\left(\begin{array}{lll}
\rho \overline{u^{\prime} u^{\prime}} & \rho \overline{u^{\prime} v^{\prime}} & \rho \overline{u^{\prime} w^{\prime}} \\
\rho \overline{v^{\prime} u^{\prime}} & \rho \overline{v^{\prime} v^{\prime}} & \rho \overline{v^{\prime} w^{\prime}} \\
\rho \overline{w^{\prime} u^{\prime}} & \rho \overline{w^{\prime} v^{\prime}} & \rho \overline{w^{\prime} w^{\prime}}
\end{array}\right)
$$

Los flujos turbulentos de calor según las direcciones $x, y$ y $z$, representados por el vector de transporte convectivo $\left(\rho c_{p} \overline{u^{\prime} T^{\prime}}, \rho c_{p} \overline{v^{\prime} T^{\prime}}, \rho c_{p} \overline{w^{\prime} T^{\prime}}\right)$ fueron también obtenidos. Para poder comparar unas zonas de la celda con otras, y en diferentes situaciones, tanto los flujos de momento como los de calor fueron normalizados de la forma siguiente:

$$
U_{i} U_{j} N=\frac{\overline{u_{i}^{\prime} u_{j}^{\prime}}}{\overline{u_{1}^{\prime} u_{1}^{\prime}}} \quad U_{i} T N=\frac{\overline{u_{i}^{\prime} T^{\prime}}}{\sqrt{\left(\overline{u_{1}^{\prime} u_{1}^{\prime}} \cdot \overline{T^{\prime} T^{\prime}}\right)}}
$$

donde $\left(u_{1}, u_{2}, u_{3}\right)$ son las componentes de la velocidad usualmente representadas por $(u, v, w)$ según el sistema de coordenadas definido.

Respecto de la intensidad de turbulencia (ITUR), que mide la agitación turbulenta del aire en el interior de la celda, se determina a partir de la covarianza, $\overline{u^{\prime} u^{\prime}}$ y, aplicando la ecuación

$$
I T U R=\frac{\sqrt{\left(\overline{u^{\prime} u^{\prime}}\right)}}{\bar{u}}
$$

Los parámetros calculados para estudiar la inercia y la flotabilidad presentes en el chorro han sido: El número Arquímedes (Ar), el número de Nusselt $(\mathrm{Nu})$ y el número de Rayleigh (Ra) (Weiran y Qingyan, 2001; Shiang-Wuu y Horng-Wen, 2007). Se incluye, también, la longitud térmica $\left(l_{m}\right)$, que aunque no es un número adimensional, juega un papel importante en el conocimiento de las condiciones para la mezcla de magnitudes físicas y los efectos de empuje hidrostático por diferencias de densidad.

El número de Arquímedes es un parámetro adimensional de gran importancia en el estudio de los chorros no isotérmicos, introducidos vertical u horizontalmente en una habitación, representa una medida relativa de las fuerzas de flotabilidad frente a las fuerzas de inercia. Se calcula, el número de Arquímedes, a la entrada de la habitación de ensayos y en tres secciones transversales a la dirección general del chorro (dirección $X$ ) mediante la ecuación

$\operatorname{Ar}(x)=\frac{g^{\prime}(x) \sqrt{A(x)}}{u(x)^{2}}$

Esta ecuación representa un número de Arquímedes local. Donde $A(x)$ es el área de la sección considerada, $u(x)$ la velocidad media del flujo en dicha sección transversal y $g{ }^{\prime}=g\left(\Delta T(x) / T_{r}\right)$ siendo 
$\Delta T(x)=T(x)-T_{r}, T(x)$ la temperatura media del chorro en la sección y $T_{r}$ una temperatura media de referencia. La longitud térmica $\left(l_{m}\right)$ (Pantokratoras, 2001) que mide la distancia, en la dirección del flujo, desde la entrada al punto donde tiene lugar la transición del flujo inicial dominante de momento a flujo de flotabilidad, se ha determinado utilizando la expresión

$l_{m}^{2}=\frac{\sqrt{A(0)} \cdot u(0)^{2}}{g^{\prime}}$

$A(0)$ es el área de la abertura de entrada, $u(0)$ la velocidad de entrada del chorro y $g$ ' tomará el valor correspondiente según lo definido en el párrafo anterior. La covarianza $\overline{u^{\prime} T^{\prime}}$ obtenida a partir de la base de datos generada en la fase experimental, permite conocer los flujos de calor de origen turbulento en la dirección del flujo (eje X) mediante la ecuación:

$Q=\rho c_{p} \overline{u^{\prime} T^{\prime}}$

siendo $\rho$ (densidad del aire $)=1.20 \mathrm{~kg} \cdot \mathrm{m}^{-3}$ y $c_{p}$ (calor específico del aire a presión constante $)=1.00$ $\times 10^{3} \mathrm{~J} \cdot \mathrm{kg}^{-1} \cdot \mathrm{K}^{-1}$.

Conocidos los flujos de calor de origen turbulento se calcula el número de Nusselt y el número de Rayleigh (Chen y Chou, 2006), en la dirección general del flujo, utilizando las ecuaciones

$(\mathrm{Nu})_{X}=\frac{\rho c_{p} H}{k\left(T-T_{r}\right)} \overline{u^{\prime} T^{\prime}}$

$(\mathrm{Ra})_{X}=\frac{g \beta \rho c_{p} H^{4}}{\alpha v k} \overline{u^{\prime} T^{\prime}}$

siendo $k$ la conductividad térmica del fluido, $H$ una altura característica, $T$ la temperatura del flujo en una sección del recinto, $T_{r}$ la temperatura de una zona de dicho recinto que se toma como referencia, $\beta$ el coeficiente de expansión térmica, $\alpha$ la difusividad térmica, y $v$ la viscosidad cinemática.

El número de Nusselt es un producto adimensional que refleja la intensidad de transporte de calor del flujo por convección, respecto al transporte de calor por difusión molecular mientras que el número de Rayleigh representa una medida de las fuerzas de flotabilidad del chorro frente a las fuerzas de inercia. En las ecuaciones (7) y (8) tanto el número de Nusselt $(\mathrm{Nu})_{X}$ como el número de Rayleigh $(\mathrm{Ra})_{X}$ reflejan, en la dirección general del flujo (dirección $X$ ), una medida de la influencia de los flujos turbulentos de calor en los fenómenos de transporte de calor asociados al chorro.

\section{RESULTADOS Y DISCUSIÓN}

Se aplica un modelo de regresión lineal múltiple y se efectúa el análisis de la varianza, mediante la aplicación informática Statgraphics, a las medidas de las magnitudes turbulentas obtenidas de la malla, que cubre toda la habitación de ensayos. Se analiza la relación de la intensidad de turbulencia (ITUR) con los flujos de momento en las direcciones transversales a la dirección general del flujo $(U V N, U W N)$, con los flujos de calor en dichas direcciones (WTN, VTN) y con la altura (Z) del sensor de velocidad.. Los flujos anteriores, que están normalizados, se definen en (2), donde se ha utilizado $(U, V, W)$ en lugar de $\left(U_{1}, U_{2}, U_{3}\right)$ por comodidad.

En el análisis de la varianza se obtiene: el coeficiente de determinación $\mathrm{R}^{2}$, el coeficiente de determinación corregido por grados de libertad $\overline{\mathrm{R}}^{2}$ y la variable aleatoria $\mathrm{F}$ de Fisher, con su correspondiente nivel de significación estadística, $\alpha$. El coeficiente de determinación corregido por 
grados de libertad es un parámetro estadístico que sustituye $\mathrm{a}^{2}$ en el caso de regresiones múltiples para evitar su aumento por el hecho de introducir nuevas variables (Peña, 2002). Las regresiones lineales múltiples y el análisis de la varianza de la variable ITUR con las variables $Z, U V N, U W N$, $W T N$ y VTN se han calculado para las posiciones $Y^{\star}=148 \mathrm{~cm}, Y^{\star}=90 \mathrm{~cm}$ e $Y^{\star}=40 \mathrm{~cm}$ del sensor de velocidad medidas respecto de la pared oeste de la habitación de ensayos, es decir, $Y^{\star}=W d-Y$, (Wd anchura de la habitación) lo que se muestra en las Tabla 1 y Tabla 2. Los resultados reflejan que en la posición $90 \mathrm{~cm}$, que es la que corresponde aproximadamente a la dirección general del chorro, existe una relación estadísticamente significativa entre las variables consideradas con un nivel de confianza del $99 \%$. El modelo explica en un $74 \%$ la variabilidad de la intensidad turbulenta con dichas magnitudes.

Tabla 1: (a) Regresión lineal múltiple de la variable ITUR frente a las variables Z, UVN, UWN y WTN

\begin{tabular}{|c|c|}
\hline (Dist. a pared oeste) & Regresiones \\
\hline $148 \mathrm{~cm}$ & ITUR $=0.43434-0.00114 \mathrm{Z}-0.01942$ UVN $-0.05032 \mathrm{UWN}+$ \\
0.01154 WTN
\end{tabular}

(b) Análisis de la varianza

\begin{tabular}{|c|c|c|c|c|}
\hline $\begin{array}{c}\mathrm{Y}^{*} \\
\text { (Dist. a pared oeste) }\end{array}$ & $\begin{array}{c}\mathrm{R}^{2} \\
\text { (Coef. Det.) }\end{array}$ & $\begin{array}{c}\overline{\mathrm{R}}^{2} \\
\left(\mathrm{R}^{2} \text { Correg.) }\right.\end{array}$ & $\begin{array}{c}\mathrm{F} \\
\text { (Fisher) }\end{array}$ & $\begin{array}{c}\alpha \\
\text { (Nivel Sig.) }\end{array}$ \\
\hline $148 \mathrm{~cm}$ & 0.66 & 0.53 & 4.91 & 0.0188 \\
\hline $90 \mathrm{~cm}$ & 0.75 & 0.65 & 7.38 & 0.0049 \\
\hline $40 \mathrm{~cm}$ & 0.64 & 0.49 & 4.40 & 0.0262 \\
\hline
\end{tabular}

Un análisis más detallado indica que el resultado del modelo mejora cuando se introduce el flujo de calor VTN (Tabla.2), es decir, la variación de la temperatura en la dirección del eje y de la habitación y en aquella posición más próxima a la pared oeste $\left(Y^{\star}=40 \mathrm{~cm}\right)$, con un valor de $\mathrm{R}^{2}$ del $81.76 \%$ y un nivel de significación estadística de la F de Fisher superior al 99\%.

Para el cálculo del número de Arquímedes a la entrada de la habitación de ensayos (posición $x=0$ ) se utilizó la ecuación (4). En el cálculo de $g^{\prime}$ en esta ecuación se tuvo en cuenta que, durante la realización de la fase experimental, la temperatura del flujo a la entrada de la habitación y las temperaturas de las paredes este y oeste presentan la misma tendencia. Por este motivo se ha tomando como temperatura, $T_{0}$, de entrada la temperatura media registrada durante la fase experimental y como temperatura de referencia, $T_{\mathrm{r}},=\mathrm{TE}$, temperatura media de la pared este. Así mismo, se ha tomado el área de la ventana de entrada del flujo como $A(0)$ y como $u(0)$ la velocidad media del flujo en dicha entrada.

Sustituyendo los valores numéricos en la ecuación, obtenemos un número de Arquímedes a la entrada de la celda para nuestro experimento de $\mathrm{Ar}=0.059$, es decir, del orden de $10^{-2}$ comprendido, por tanto, en el intervalo $0<\mathrm{Ar}<0.2$ que corresponde a una extensión lateral del chorro despreciable $\mathrm{y}$, en consecuencia, a una influencia de las fuerzas de flotabilidad, también, 
despreciable. Una vez estimado Ar a la entrada se determina, en tres secciones transversales a la dirección general del chorro (número de Arquímedes local) y para tres niveles de la habitación de ensayos. Las secciones se han tomado respecto de la ventana, de entrada del flujo, a $x=1.50 \mathrm{~m}, x=$ $2.50 \mathrm{~m}$ y de $x=3.50 \mathrm{~m}$. En cuanto a los niveles corresponden a $z=1.40 \mathrm{~m}, z=0.70 \mathrm{~m}$ y $z=0.43 \mathrm{~m}$ (Tabla.1).

Tabla 2: (a) Regresión lineal múltiple de la variable ITUR frente a las variables $Z, U V N$, UWN, WTN y VTN

\begin{tabular}{|c|c|}
\hline $\begin{array}{c}\mathrm{Y}^{*} \\
\text { (Dist. a pared oeste) }\end{array}$ & Regresiones \\
\hline $148 \mathrm{~cm}$ & $\begin{array}{c}\text { ITUR }=0.48042-0.00171 \mathrm{Z}-0.02657 \text { UVN }-0.06453 \text { UWN + } \\
0.02447 \text { WTN }-0.10864 \text { VTN }\end{array}$ \\
\hline $90 \mathrm{~cm}$ & $\begin{array}{c}\text { ITUR }=0.74869-0.00502 \mathrm{Z}+0.72008 \mathrm{UVN}+0.63241 \mathrm{UWN}+0.05893 \\
\text { WTN }-0.00704 \text { VTN }\end{array}$ \\
\hline $40 \mathrm{~cm}$ & $\begin{array}{c}\text { ITUR }=0.50737-0.00110 \mathrm{Z}-0.06616 \text { UVN }+0.05076 \text { UWN + } \\
+0.36722 \text { WTN }-0.20327 \text { VTN }\end{array}$ \\
\hline
\end{tabular}

(b) Análisis de la varianza

\begin{tabular}{|c|c|c|c|c|}
\hline $\begin{array}{c}\mathrm{Y}^{*} \\
\text { (Dist. a pared Oeste) }\end{array}$ & $\begin{array}{c}\mathrm{R}^{2} \\
\text { (Coef. Det.) }\end{array}$ & $\begin{array}{c}\overline{\mathrm{R}}^{2} \\
\left(\mathrm{R}^{2} \text { Correg.) }\right.\end{array}$ & $\begin{array}{c}\mathrm{F} \\
\text { (Fisher) }\end{array}$ & $\begin{array}{c}\alpha \\
\text { (Nivel Sig.) }\end{array}$ \\
\hline $148 \mathrm{~cm}$ & 0.80 & 0.68 & 7.00 & 0.0062 \\
\hline $90 \mathrm{~cm}$ & 0.75 & 0.61 & 5.32 & 0.0151 \\
\hline $40 \mathrm{~cm}$ & 0.82 & 0.72 & 8.07 & 0.0038 \\
\hline
\end{tabular}

La Tabla.3, muestra cómo los niveles presentan un comportamiento similar del número de Arquímedes respecto a su variación con la distancia a la ventana de entrada del flujo. También, observamos un aumento Ar hacia los niveles más bajos, es decir, según nos aproximamos al suelo de la habitación. Analizando los resultados se deduce que la distribución del flujo en el interior de la habitación es debida, por una parte, a los fuertes efectos dinámicos de dicho flujo a la entrada que permite que el chorro penetre en el interior y que pueda llegar a alcanzar niveles próximos al suelo y, por otra parte, es debida a la importancia que van adquiriendo las fuerzas de flotabilidad del chorro, en su paso a través de dicha habitación, que le permite expandirse lateral y verticalmente a la dirección general del mismo.

Tabla 3: Número de Arquímedes en el interior de la celda

\begin{tabular}{|c|c|c|c|}
\hline $\mathrm{Z}_{Z}^{X}$ & 150 & 250 & 350 \\
\hline 140 & 1.46 & 3.84 & 6.44 \\
\hline 70 & 3.55 & 4.28 & 4.00 \\
\hline 43 & 7.29 & 24.47 & 29.03 \\
\hline
\end{tabular}


A partir de la ec. (5) se ha obtenido un valor de la longitud térmica $\left(l_{m}\right)$ de $2.30 \mathrm{~m}$ que corresponde, prácticamente, a la mitad de la longitud de la habitación de ensayo. Es decir, hacia la mitad de la habitación el chorro empieza a perder su carácter inercial, comienzan a predominar, por tanto, las fuerzas de flotabilidad coincidiendo con la región de desarrollo completo de la turbulencia. Finalmente, se calcula el número de Nusselt $(\mathrm{Nu})_{x}$ y el número de Rayleigh $(\mathrm{Ra})_{x}$, en la dirección general del flujo (dirección $X$ ), mediante la ec. (7) y la ec. (8) para las mismas secciones consideradas en la determinación del número de Arquímedes y para los mismos niveles. En las Figuras 2 y 3 , se muestran las distribuciones de $(\mathrm{Nu})_{x}$ y $(\mathrm{Ra})_{x}$, según el eje $Y$. Los valores de $y$ se presentan sin dimensiones dividiendo por la anchura de la ventana.

La distribución del parámetro $(\mathrm{Nu})_{x}$ (Fig.2) presenta tendencias semejantes tanto en el nivel $z=1.40$ $\mathrm{m}$ como $\mathrm{z}=0.43 \mathrm{~m}$, observando además, que estos parámetros varían, en general, muy poco y de forma uniforme en la dirección de la pared oeste de la celda. No obstante, se observa la existencia de un máximo del parámetro $(\mathrm{Nu})_{X}$ bastante llamativo, en un punto del nivel correspondiente $\mathrm{a} z=$ $1.40 \mathrm{~m}$ situado hacia la mitad de la habitación de ensayos y en la dirección general del chorro. Este comportamiento del número Nusselt es debido a que la altura de $1.40 \mathrm{~m}$ representa, por un lado, una posición ligeramente por encima de la dirección general de dicho chorro, lo que justificaría el valor máximo de $(\mathrm{Nu})_{x}$, y por otro lado, una posición alejada del orificio de salida del flujo y, en consecuencia, de su trayectoria.
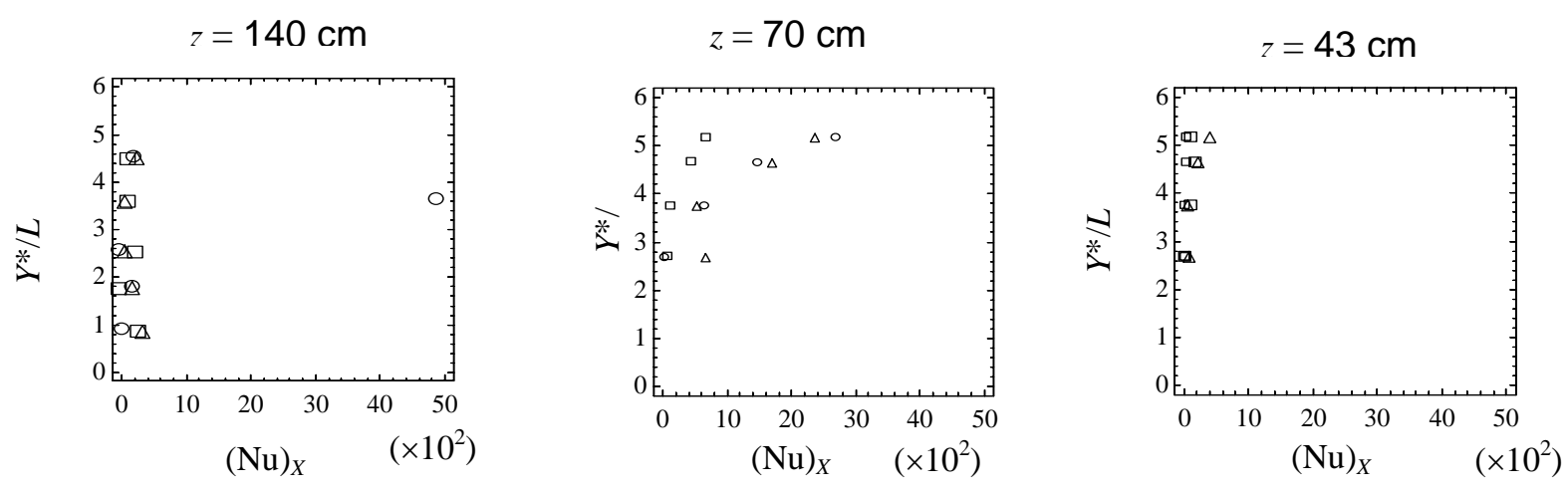

Fig. 2: Perfil de $(\mathrm{Nu})_{X}$ para tres secciones transversales al eje $X: \square 150 \mathrm{~cm}$; $\bigcirc 250$ $\mathrm{cm} ; \triangle 350 \mathrm{~cm}$.
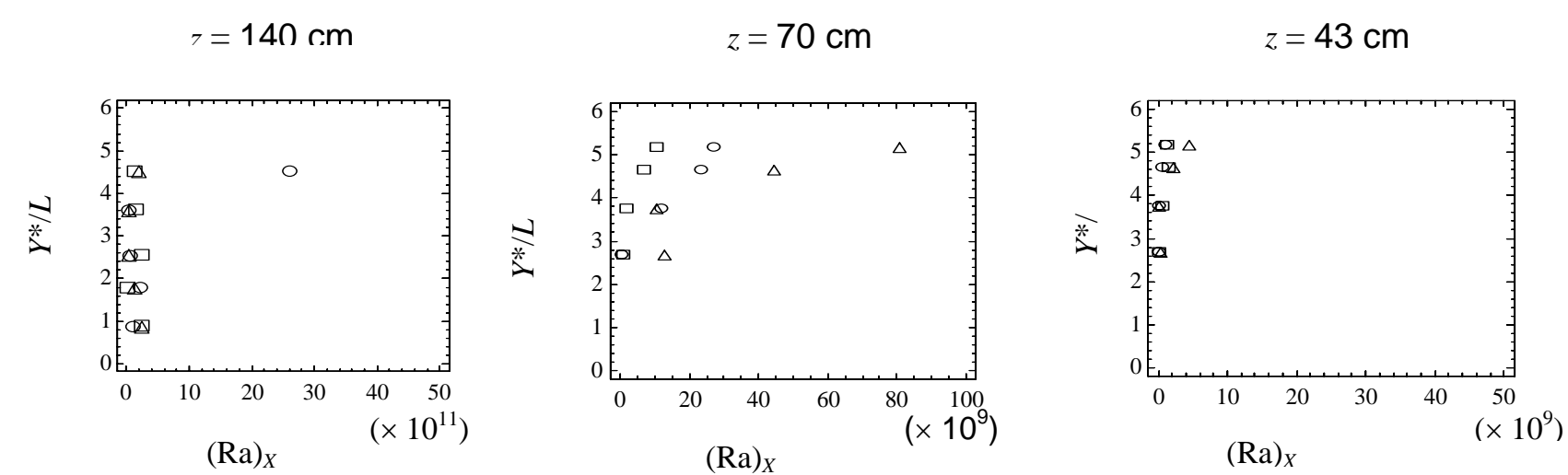

Fig. 3: Perfil de $(\mathrm{Ra})_{X}$ para tres secciones transversales al eje $X: \square 150 \mathrm{~cm}$; $\bigcirc 250$ $\mathrm{cm} ; \triangle 350 \mathrm{~cm}$.

Los perfiles de $(\mathrm{Nu})_{x}$ en el nivel $z=0.70$ m reflejan, un aumento significativo de este parámetro hacia la mitad de la habitación de ensayos y hacia la pared oeste que coincide con el incremento de intensidad de turbulencia observada por niveles entre la altura de la dirección general del chorro y el orificio de salida, con los máximos de intensidad de turbulencia observados próximos al centro de la 
habitación, según nos aproximamos a la pared oeste, y con la expansión lateral y vertical del chorro tal y como se desprende del estudio del número de Arquímedes. El comportamiento de este parámetro, en este nivel se atribuye a que mide las propiedades físicas en una zona intermedia entre la dirección general del chorro y la altura del orificio de salida del flujo y, por tanto, influenciados por los flujos de calor de origen turbulentos asociados al chorro. Así mismo, la Figura.3 muestra las distribuciones correspondientes al número de Rayleigh. Los resultados indican que el orden de magnitud del número Rayleigh $\left(\geq 10^{8}\right)$, en las direcciones consideradas, es el que corresponde a flujos turbulentos en el interior de un determinado recinto. Para los niveles considerados, se aprecia que el comportamiento del parámetro $(\mathrm{Ra})_{x}$ concuerda con el comportamiento de $(\mathrm{Nu})_{x}$ (Fig.2 y Fig.3), lo que nos permite confirmar que existe una dependencia del número de Nusselt con el número de Rayleigh en este trabajo.

\section{CONCLUSIONES}

A partir del análisis de los resultados, se extraen las siguientes conclusiones relevantes: a) La aplicación del modelo de regresión lineal múltiple con los parámetros y magnitudes descritos anteriormente, explica en un $81,76 \%$ la variabilidad de la intensidad turbulenta con los flujos turbulentos y Z, con un nivel de confianza estadística superior al $99 \%$. b) La distribución del número de Arquímedes indica que la efectos dinámicos del flujo son más importantes a la entrada y los efectos de flotabilidad prevalecen a partir de la mitad de la habitación lo que le permite expandirse lateral y verticalmente alcanzando niveles próximos al suelo, c) La determinación del número de Nusselt refleja la aparición de un proceso de transferencia de calor de origen turbulento en los niveles comprendidos entre la altura media del chorro y el orificio de salida. Este proceso coincide con el incremento de intensidad de turbulencia observado en los mismos niveles, en zonas próximas al centro de la habitación y hacia la pared oeste. d) El número de Rayleigh presenta un comportamiento concordante con el número de Nusselt, en niveles entre la altura del chorro y el orificio de salida, lo que facilita las condiciones para que aparezca un importante proceso de transferencia de calor de origen turbulento.

\section{NOMENCLATURA}

$\begin{array}{ll}\text { AHC } & \text { Anemómetro de hilo caliente } \\ \mathrm{AS} & \text { Anemómetro sónico } \\ c_{p} & \text { Calor específico a presión constante } \\ X, Y, Z & \text { Coordenadas cartesianas } \\ Y^{\star} & \text { Distancia a la pared oste }(\mathrm{m}) \\ u_{i}{ }^{\prime} & \text { Fluctuación de Velocidad }\left(\mathrm{m} \mathrm{s}^{-1}\right) \\ \rho c_{p} \overline{u_{i}{ }^{\prime} T^{\prime}} & \text { Flujo de calor } \\ \rho \overline{u_{i}{ }^{\prime} v_{j}{ }^{\prime}} & \text { Flujo de momento } \\ U_{i} T N & \text { Flujos de calor normalizados } \\ U_{i} U_{j} N & \text { Flujos de momento normalizados } \\ U V N, U W N & \text { Flujos trasversales normalizados } \\ I T U R & \text { Intensidad de turbulencia } \\ L, W d, H & \text { Largo, ancho, alto en la celda (m) } \\ l_{m} & \text { Longitud térmica (m) } \\ \mathrm{Ar} & \text { Número de Arquímedes } \\ \mathrm{Nu} & \text { Número de Nusselt } \\ \mathrm{Ra} & \text { Número de Rayleigh } \\ S & \text { Sección de la ventana }\left(\mathrm{m}^{2}\right) \\ \mathrm{T}_{0} & \left.\text { Temperatura en la ventana ( }{ }^{\circ} \mathrm{C}\right) \\ \mathrm{TE} & \text { Temperatura pared este }\left({ }^{\circ} \mathrm{C}\right) \\ \mathrm{TC} & \text { Termopares } \\ V_{\max } & \text { Velocidad máxima } \\ V & \text { Velocidad media del flujo } \\ \rho & \text { Densidad del aire }\end{array}$




\section{REFERENCIAS}

Chen, H. y J. Chou; Investigation of natural-convection heat transfer coefficient on a vertical square fin of finned-tube heat exchanger. International J. of Heat and Mass Transfer: 49, 3034-3044, (2006)

Costa, J.J., L.A. Oliveira y D. Blay; Test of several versions the $k-\varepsilon$ type turbulence modelling of internal mixed convection flows. International J. of Heat and Mass Transfer: 42, 4391-4409 (1999).

Costa, V.A.F; Unification of the streamline, heatline and massline methods for the visualization of twodimensional transport phenomena. International J. of Heat and Mass Transfer: 42, 27-33 (1999).

Etheridge, D. y M. Sandberg; Building Ventilation: Theory and Measurement. Ed. John Wiley and Sons (1996).

Dubovsky, V., G. Ziskind; S.Druckman; E. Mashka; Y. Weiss y R. Letan, Natural convection inside ventilated enclosure heated by downward-facing plate: experiments and numerical simulations. International of Journal Heat and Mass Transfer. 44, 3155-3168 (2001).

Kotsovinos, N.E. y P.B Angelidis; The momentum flux in turbulent submerged jets. J. Fluid Mech.: 229, 453-470 (1991).

Kruse N. y R. von Rohr; Structure of turbulent heat flux in a flow over a heated wavy wall. International Journal of Heat and Mass Transfer. 49, 3514-3529 (2006).

Launder, B.E. y W. Rodi; The turbulent wall jet-measurements and modelling. Ann. Rev. Fluid Mech.: 15, 429-459 (1983).

Pantokratoras, A.; Effect of ambient temperature on vertical turbulent buoyant water jets. International J. of Heat and Mass Transfer: 44, 1889-1898 (2001).

Peña Sánchez de Rivera, D.; Regresión y Diseño de Experimentos. Ed. Alianza Editorial. (2002)

Shiang-Wuu P. y W. Horng-Wen W.; Buoyancy-aided/opposed convection heat transfer for unsteady turbulent flow across a square cylinder in a vertical channel. International $\mathrm{J}$. of Heat and Mass Transfer: 50 3701-3717 (2007).

Viñas, C.; Análisis del Flujo Turbulento en el Interior de una Celda "CESPA" Mediante Aplicación de la Teoría de Capa Límite. Tesis Doctoral. Univ. Complutense de Madrid (2003).

Viñas, C. y G. Maqueda; A Spectral Analysis Method to Study Indoors Flows. Actas EGU General Assembly, Vienna (April, 2005).

Weiran X. y C. Qingyan; A two-layer turbulence model for simulating indoor airflow. Energy and Buildings: 33, 613-625 (2001). 